\title{
ON THE LEIBNIZ CONGRUENCES
}

\author{
JOSEP M. FONT \\ Department of Logic, History and Philosophy of Science \\ Faculty of Mathematics, University of Barcelona \\ Gran Via 585, 08007 Barcelona, Spain \\ E-mail:FONT@CERBER.UB.ES
}

\begin{abstract}
The aim of this paper is to discuss the motivation for a new general algebraic semantics for deductive systems, to introduce it, and to present an outline of its main features. Some tools from the theory of abstract logics are also introduced, and two classifications of deductive systems are analysed: one is based on the behaviour of the Leibniz congruence (the maximum congruence of a logical matrix) and the other on the behaviour of the Frege operator (which associates to every theory the interderivability relation modulo the theory). For protoalgebraic deductive systems the class of algebras associated in general turns out to be the class of algebra reducts of reduced matrices, which is the algebraic counterpart usually considered for this large class of deductive systems; but in the general case the new class of algebras shows a better behaviour.
\end{abstract}

The original purpose of this talk $\left(^{1}\right)$ was twofold: To survey some of the ideas that have been present in some of the research in Algebraic Logic done by some of the people in Barcelona over the years, and to present a proposal of a very general definition of what constitutes the algebraic counterpart of a deductive system; by this I hoped to convey my point of view on Algebraic Logic, or at least some of its facets, and to present a number of research problems and proposals. Actually the first goal proved to be too ambitious for the occasion, and I decided to focus

1991 Mathematics Subject Classification: Primary 03B22; Secondary 03B20, 45, 46, 50, 55, 03G10, 25.

Key words and phrases: algebraic logic, abstract logic, closure operator, Leibniz congruence, logical matrices, selfextensional logic, algebraizable logic, protoalgebraic logic, deductive system, Gentzen calculus.

Supported by grants PB90-0465-C02-01 of Spanish DGICYT and BV91/L/1-237 of CIRIT, Generalitat de Catalunya. The author would also like to thank Ramon Jansana and Ventura Verdú for their comments on a draft of this paper.

The paper is in final form and no version of it will be published elsewhere.

$\left({ }^{1}\right)$ Given on October 8th, 1991, at the Banach Center, Warsaw. 
on the motivation for this new proposal, due to Ramon Jansana and myself, by trying to present our first results as fitting into a broader, coherent framework, relating them to other people's work, as far as this is possible. To this end I have chosen to organize this talk around one main concept, namely that of the Leibniz congruence, a term coined by Wim Blok and Don Pigozzi in [4] to denote a rather old concept (the greatest congruence of a logical matrix) and which I will use here to cover also a natural extension of this notion. Hence the plural in the title.

The Leibniz operator. Let me begin by recalling some terms and fixing some notations; detailed definitions of the most basic concepts can be found for instance in [4], [11], [15] or [56] (but note some differences in terminology and/or in notation).

I will deal only with deductive systems (also called propositional logics, or simply logics), presented as $\mathcal{S}=\left\langle\mathfrak{F} \mathfrak{m}, \mathbf{C}_{\mathcal{S}}\right\rangle$ or $\mathcal{S}=\left\langle\mathfrak{F m}, \vdash_{\mathcal{S}}\right\rangle$ or $\langle\mathfrak{F m}, T h(\mathcal{S})\rangle$, where:

$\mathfrak{F m}=\langle F m, \ldots\rangle$ is the algebra of (propositional) formulas of some (fixed) algebraic type; the variables (i.e., the generators of the absolutely free algebra $\mathfrak{F m})$ will be denoted by $p, q, r, \ldots$;

$\mathbf{C}_{\mathcal{S}}$ is a finitary and structural closure operator on $\mathrm{Fm}$; traditionally the associated relation is denoted by $\vdash_{\mathcal{S}}$, or simply by $\vdash$, thus $\Gamma \vdash \varphi$ means $\varphi \in$ $\mathbf{C}_{\mathcal{S}}(\Gamma) ;$ and

$T h(\mathcal{S})$ is the associated closure system (the theories of $\mathcal{S}$ ).

Any kind of algebraic models of a deductive system must be built on algebras $\mathfrak{A}=\langle A, \ldots\rangle$ similar to $\mathfrak{F} \mathfrak{m}$; when dealing with any of these, we will be specially concerned with $\operatorname{Con}(\mathfrak{A})$, the set (lattice) of all congruences of $\mathfrak{A}$; and for any class $\mathbb{K}$ of algebras of the same type, with the set of $\mathbb{K}$-congruences of $\mathfrak{A}, \operatorname{Con}_{\mathbb{K}}(\mathfrak{A})=$ $\{\theta \in \operatorname{Con}(\mathfrak{A}): \mathfrak{A} / \theta \in \mathbb{K}\}$. The simplest kind of algebraic models of a deductive system one can consider on any of these algebras are the $\mathcal{S}$-matrices, or matrices for $\mathcal{S}$, which are pairs $\langle\mathfrak{A}, F\rangle$ where $F \subseteq A$ is such that for any $\Gamma \subseteq F m, \varphi \in F m$,

$$
\Gamma \vdash \mathcal{S} \varphi \Rightarrow \forall h \in \operatorname{Hom}(\mathfrak{F m}, \mathfrak{A}), h(\Gamma) \subseteq F \Rightarrow h(\varphi) \in F .
$$

Then $F$ is called an $\mathcal{S}$-filter. The class of all $\mathcal{S}$-matrices is denoted by $\operatorname{Matr}(\mathcal{S})$; and for each algebra $\mathfrak{A}$ we consider the set of $\mathcal{S}$-filters on $\mathfrak{A}: \mathcal{F}_{\mathfrak{A}}^{\mathcal{S}}=\{F \subseteq A:\langle\mathfrak{A}, F\rangle$ is a matrix for $\mathcal{S}$ \}.

Some algebraic concepts associated with a logical matrix, however, do not depend on it being a matrix for any particular deductive system. For instance, if $F \subseteq A$, a congruence $\theta \in \operatorname{Con}(\mathfrak{A})$ is said to be compatible with $F$, or to be a matrix congruence of $\langle\mathfrak{A}, F\rangle$, when $\forall a, b \in A$, if $\langle a, b\rangle \in \theta$ then $(a \in F \Leftrightarrow b \in F)$; the set of all these congruences is denoted by $\operatorname{Con}(\langle\mathfrak{A}, F\rangle)$, and always has a maximum. Using the terminology introduced in [4], by the Leibniz operator I understand the mapping $F \mapsto \Omega_{\mathfrak{A}}(F)$, where

$$
\Omega_{\mathfrak{A}}(F)=\max \operatorname{Con}(\langle\mathfrak{A}, F\rangle)
$$


is called the Leibniz congruence of $F$, or of $\langle\mathfrak{A}, F\rangle$; the motivation for giving it this name - related to G. W. Leibniz's second-order definition of identity - is very clearly explained in [4, pp. $10 \mathrm{ff}]$, and is related to the following interesting (and well-known) characterization:

$$
\begin{aligned}
\langle a, b\rangle \in \Omega_{\mathfrak{A}}(F) \Leftrightarrow \varphi^{\mathfrak{A}}(h(a / p)) \in F \Leftrightarrow & \varphi^{\mathfrak{A}}(h(b / p)) \in F \\
& \forall \varphi \in F m, \forall h \text { interpretation in } \mathfrak{A}
\end{aligned}
$$

(an interpretation in $\mathfrak{A}$ is just an $h \in \operatorname{Hom}(\mathfrak{F m}, \mathfrak{A})$; and $h(a / p)$ denotes the interpretation obtained after modifying $h$ by giving $p$ the value $a$ ). Here and in other places, when dealing with the formula algebra the subscript indicating the algebra will be omitted, that is, $\Omega=\Omega_{\mathfrak{F m}}$. The matrices having only one matrix congruence are called reduced (or sometimes simple, or factorized):

$$
\operatorname{Matr}^{*}(\mathcal{S})=\left\{\langle\mathfrak{A}, F\rangle \in \operatorname{Matr}(\mathcal{S}): \Omega_{\mathfrak{A}}(F)=\Delta_{A}\right\} .
$$

The most usual algebraic counterpart of a deductive system is the class of algebra reducts of the reduced matrices:

$$
\operatorname{Alg}^{*}(\mathcal{S})=\left\{\mathfrak{A}: \exists F \subseteq A \text { with }\langle\mathfrak{A}, F\rangle \in \operatorname{Matr}^{*}(\mathcal{S})\right\}
$$

(this notation is an extended use of that introduced in [40]). However, we will see that in some cases there is another class of algebras which better deserves such name.

The Leibniz operator, specially $\Omega$, is very interesting. It is one of the fundamental tools of Blok and Pigozzi's monographs [4]-[7], which contain some deep results about it. It turns out that several outstanding classes of deductive systems, although originally defined in different forms, have been characterized by the behaviour of this operator on their filters. Thus we have:

- $\mathcal{S}$ is protoalgebraic $\Leftrightarrow \forall \mathfrak{A}, \Omega_{\mathfrak{A}}$ is order-preserving on $\mathcal{F} i_{\mathfrak{A}}^{\mathcal{S}}$, i.e., if $F, G \in \mathcal{F} i_{\mathfrak{A}}^{\mathcal{S}}$ then $F \subseteq G$ implies $\Omega_{\mathfrak{A}}(F) \subseteq \Omega_{\mathfrak{A}}(G)$ [2, Theorem 2.4].

- $\mathcal{S}$ is equivalential (or weakly congruential [7]) $\Leftrightarrow$ All mappings $\Omega_{\mathfrak{A}}$ are, so to speak, "uniformly analytically definable", i.e., there is $E(p, q) \subseteq F m$ such that $\forall\langle\mathfrak{A}, F\rangle \in \operatorname{Matr}(\mathcal{S}), \forall a, b \in A,\langle a, b\rangle \in \Omega_{\mathfrak{A}}(F) \Leftrightarrow E^{\mathfrak{A}}(a, b) \subseteq F[12$, Theorem I.11].

- $\mathcal{S}$ is finitely equivalential (or congruential [7]) $\Leftrightarrow \mathcal{S}$ is equivalential with a finite $E(p, q)$. Then it can be shown that all the possible sets of equivalence formulas $E(p, q)$ can be taken finite.

- $\mathcal{S}$ is algebraizable with respect to a quasivariety $\mathbb{K}$ (called its equivalent quasivariety semantics) $\Leftrightarrow \forall \mathfrak{A}, \Omega_{\mathfrak{A}}$ is an isomorphism between the lattices $\mathcal{F} i_{\mathfrak{A}}^{\mathcal{S}}$ and $\operatorname{Con}_{\mathbb{K}}(\mathfrak{A})[4$, Theorem 5.1].

These four classes are increasingly smaller. The deductive systems belonging to them have been studied by several people; see specially the works by Czelakowski, Dziobiak, Blok and Pigozzi quoted in the References section, and more particularly [7], where this hierarchy is deeply analysed in a general framework. They have interesting algebraic and syntactical characterizations, their matrices behave 
reasonably well, and the relationship between the deductive system and the class of matrices is good, and becomes better as the class gets smaller. The extreme case is that of algebraizable logics, which present the strongest relationship so far described between the deduction in $\mathcal{S}$ and the equational consequence $\vDash_{\mathbb{K}}$ relative to the class $\mathbb{K}$ defined as follows: If $\Xi \subseteq F m \times F m$ is a set of equations and $\varphi \approx \psi$ is an equation, then

$$
\begin{array}{r}
\Xi \vDash_{\mathbb{K}} \varphi \approx \psi \Leftrightarrow \text { If } \xi^{\mathfrak{A}}(h)=\eta^{\mathfrak{A}}(h) \text { for all } \xi \approx \eta \in \Xi \text { then } \varphi^{\mathfrak{A}}(h)=\psi^{\mathfrak{A}}(h), \\
\text { for any interpretation } h \text { on any } \mathfrak{A} \in \mathbb{K} .
\end{array}
$$

This defines a closure operator on the set of equations, which should not be confused with the stronger one associated with ordinary equational logic (for more information on $\vDash_{\mathbb{K}}$, see [5]-[7], where it is treated as a "2-dimensional deductive system"). In algebraizable logics the consequence relations $\vdash_{\mathcal{S}}$ and $\models_{\mathbb{K}}$ are strongly equivalent, namely they are so by means of two elementary definable finite translations, from formulas into equations and backwards; I am omitting the details, as these can be found in the papers just quoted. It will however be useful to recall here the following properties:

- If $\mathcal{S}$ is algebraizable with respect to a quasivariety $\mathbb{K}$, then $\mathbb{K}=A l g^{*}(\mathcal{S})$ [4, Corollary 5.3], and the Leibniz operator $\Omega$ is an isomorphism between the lattices $T h(\mathcal{S})$ and $T h\left(\vDash_{\mathbb{K}}\right)$.

- $\mathcal{S}$ is algebraizable if and only if on $T h(\mathcal{S}), \Omega$ is injective and preserves unions of directed families of theories [4, Theorem 4.2].

Note that the definition I used before to introduce the notion of "being algebraizable with respect to $\mathbb{K}$ " is in fact an equivalent characterization of the general concept, only valid if $\mathbb{K}$ is a quasivariety; we see that in this case $\mathbb{K}$ is uniquely determined by the deductive system, namely it equals $\operatorname{Alg}^{*}(\mathcal{S})$. See Blok and Pigozzi's papers, specially [4] and [7], for the general notions and for other nice characterizations (including a sound motivation for the alternative names of two of the classes of deductive systems defined above).

The extended Leibniz operator and the Frege operator. Observe that since $\Omega_{\mathfrak{A}}(\emptyset)=A \times A$, the only protoalgebraic logics without theorems are the so-called almost inconsistent ones, those with $T h(\mathcal{S})=\{\emptyset, F m\}$. Thus, all interesting logics without theorems fall outside these classes; this includes all logics $\vdash_{\mathbf{V}}$ associated with varieties $\mathbf{V}$ in [41]; and there are also other interesting cases, with or without theorems (see later). I would like to show some ways to study them algebraically and to associate with them in a canonical way a class of algebras which in some sense behaves like the equivalent quasivariety semantics for algebraizable logics and like the class $\operatorname{Alg}^{*}(\mathcal{S})$ for the protoalgebraic ones.

The role played until now by the theory of logical matrices should be played in this approach by the theory of abstract logics, begun twenty years ago by a 
group of researchers under the direction of Roman Suszko (see $[9,10])$; so I will recall the minimal definitions and notations needed here.

According to [10, Definition V.1], an abstract logic is a pair $\mathbb{L}=\langle\mathfrak{A}, \mathbf{C}\rangle$ or $\mathbb{L}=$ $\langle\mathfrak{A}, \mathcal{C}\rangle$ where $\mathfrak{A}$ is an arbitrary algebra (of suitable type), $\mathbf{C}$ is a closure operator on $A$ and $\mathcal{C}$ is its associated closure system. Given the duality between closure operators and closure systems, I will use indistinctly both forms of expressing an abstract logic. This concept is a generalization of the concept of a deductive system as presented before, but at the same time it is a generalization of the concept of logical matrix, and also covers the semantical notion of generalized or ramified matrix (see [56]). It embodies in a single mathematical object the algebraic or grammatical structure $\mathfrak{A}$ and the logical (semantical or syntactical) one, i.e., the deductive structure, given by $\mathbf{C}$ and/or $\mathcal{C}$. By allowing arbitrary algebras we also cover "deductive" structures of a purely algebraic nature, like those given by the operators of filter-generation in lattices and other structures.

Two relationships between abstract logics will be used later on. The first one is extension: Given two abstract logics $\mathbb{L}_{1}=\left\langle\mathfrak{A}, \mathbf{C}_{1}\right\rangle$ and $\mathbb{L}_{2}=\left\langle\mathfrak{A}, \mathbf{C}_{2}\right\rangle$ over the same algebra $\mathfrak{A}$, we say that $\mathbb{L}_{1}$ is weaker or smaller than $\mathbb{L}_{2}$, or that $\mathbb{L}_{2}$ is an extension of $\mathbb{L}_{1}$, when $\forall X \subseteq A, \mathbf{C}_{1}(X) \subseteq \mathbf{C}_{2}(X)$; it is equivalent to say that $\mathcal{C}_{2} \subseteq \mathcal{C}_{1}$, that is, that $\mathcal{C}_{1}$ is "finer" than $\mathcal{C}_{2}$. This is an ordering relation in all abstract logics over the same algebra, represented if necessary by $\mathbb{L}_{1} \leq \mathbb{L}_{2}$ (note that this order is the dual of the inclusion relation among closure systems). The second one is projective generation; here I will only mention a particular case: Given any two abstract logics $\mathbb{L}_{1}=\left\langle\mathfrak{A}_{1}, \mathbf{C}_{1}\right\rangle$ and $\mathbb{L}_{2}=\left\langle\mathfrak{A}_{2}, \mathbf{C}_{2}\right\rangle$, we say that $h \in \operatorname{Hom}\left(\mathfrak{A}_{1}, \mathfrak{A}_{2}\right)$ is a bilogical morphism from $\mathbb{L}_{1}$ to (or onto) $\mathbb{L}_{2}$ when $h$ is an epimorphism and $\mathcal{C}_{1}=\left\{h^{-1}(T): T \in \mathcal{C}_{2}\right\}$. This is a very strong relationship between two abstract logics, since then $h$ induces an isomorphism between the lattices $\mathcal{C}_{1}$ and $\mathcal{C}_{2}$, and we also have $\mathbf{C}_{1}=h^{-1} \circ \mathbf{C}_{2} \circ h$ and $\mathbf{C}_{2}=h \circ \mathbf{C}_{1} \circ h^{-1}$.

It is very convenient to associate with any abstract logic $\mathbb{L}=\langle\mathfrak{A}, \mathbf{C}\rangle$ the following relation, which I will call the Frege relation:

$$
\begin{aligned}
\Lambda(\mathbf{C}) & =\{\langle a, b\rangle \in A \times A: \mathbf{C}(a)=\mathbf{C}(b)\} \\
& =\{\langle a, b\rangle \in A \times A: \forall F \in \mathcal{C}, a \in F \Leftrightarrow b \in F\} .
\end{aligned}
$$

Using it, the so-called logical congruences or $\mathbb{L}$-congruences, an extension of the idea of matrix congruence, can be defined as follows:

$$
\begin{aligned}
\operatorname{Con}(\mathbb{L}) & =\{\theta \in \operatorname{Con}(\mathfrak{A}): \theta \subseteq \Lambda(\mathbf{C})\} \\
& =\{\theta \in \operatorname{Con}(\mathfrak{A}): \forall a, b \in A,\langle a, b\rangle \in \theta \Rightarrow \mathbf{C}(a)=\mathbf{C}(b)\}
\end{aligned}
$$

and in view of (2) it results that

$$
\operatorname{Con}(\mathbb{L})=\bigcap\{\operatorname{Con}(\langle\mathfrak{A}, F\rangle): F \in \mathcal{C}\} .
$$

Any $\theta \in \operatorname{Con}(\mathfrak{A})$ determines the so-called logical quotient $\mathbb{L} / \theta=\langle\mathfrak{A} / \theta, \mathcal{C} / \theta\rangle$, where $\mathcal{C} / \theta=\left\{T \subseteq A / \theta: \pi^{-1}(T) \in \mathcal{C}\right\}$ and $\pi: A \rightarrow A / \theta$ is the canonical 
projection; if in addition $\theta \in \operatorname{Con}(\mathbb{L})$ then $\mathcal{C} / \theta=\{F / \theta: F \in \mathcal{C}\}$, and $\pi$ is a bilogical morphism from $\mathbb{L}$ onto $\mathbb{L} / \theta$, by [10, Theorem VIII.6].

On the other hand, although the equivalence relation $\Lambda(\mathbf{C})$ may not be a congruence, the set $\operatorname{Con}(\mathbb{L})$ always has a maximum $\widetilde{\Omega}_{\mathfrak{A}}(\mathbb{L}) ;$ I think it can be called the (extended) Leibniz congruence of $\mathbb{L}$ because of the similarity in purpose and in definition to the matrix case, and also because by (3) one can prove that

$$
\widetilde{\Omega}_{\mathfrak{A}}(\mathbb{L})=\max \operatorname{Con}(\mathbb{L})=\bigcap\left\{\Omega_{\mathfrak{A}}(F): F \in \mathcal{C}\right\} .
$$

Since this congruence is actually associated with the closure system $\mathcal{C}$ as a family of subsets of $A$, sometimes I will write $\widetilde{\Omega}_{\mathfrak{A}}(\mathcal{C})$ instead of $\widetilde{\Omega}_{\mathfrak{A}}(\mathbb{L})$; and $\widetilde{\Omega}_{\mathfrak{A}}$ as a mapping can be called the (extended) Leibniz operator. From (4), (1) and (2), it follows at once that

$$
\begin{array}{r}
\langle a, b\rangle \in \widetilde{\Omega}_{\mathfrak{A}}(\mathcal{C}) \Leftrightarrow\left\langle\varphi^{\mathfrak{A}}(h(a / p)), \varphi^{\mathfrak{A}}(h(b / p))\right\rangle \in \Lambda(\mathbf{C}) \\
\forall \varphi \in F m, \forall h \text { interpretation in } \mathfrak{A} .
\end{array}
$$

An abstract logic is called reduced or simple if and only if it has only one logical congruence, that is, if and only if $\widetilde{\Omega}_{\mathfrak{A}}(\mathbb{L})=\Delta_{A}$; they are the analog of reduced matrices, and, as we shall see, they play a similar role in the algebraization of non-protoalgebraic logics.

Given an abstract logic $\mathbb{L}=\langle\mathfrak{A}, \mathbf{C}\rangle$ we can consider the following operator on subsets of $A$, which I will call the Frege operator:

$$
F \subseteq A \mapsto \Lambda_{\mathbf{C}}(F)=\{\langle a, b\rangle \in A \times A: \mathbf{C}(F, a)=\mathbf{C}(F, b)\} .
$$

A characterization of the finitarity of $\mathbf{C}$ in terms of the mapping $\Lambda_{\mathbf{C}}$ will be given in Proposition 7. Sometimes it will be convenient to view it as a particular case of the ordinary Frege relation; for this it suffices to associate with any $F \subseteq A$ the following extension of $\mathbb{L}$ by $F$ :

$$
\mathbb{L}^{F}=\left\langle\mathfrak{A}, \mathcal{C}^{F}\right\rangle \quad \text { where } \quad \mathcal{C}^{F}=\{G \in \mathcal{C}: G \supseteq F \text { or } G=\emptyset\} .
$$

Note that if $\emptyset \notin \mathcal{C}$ then $\mathbb{L}^{F}$ is an "axiomatic extension" of $\mathbb{L}$ in the ordinary sense, while if $\emptyset \in \mathcal{C}$ then for any $F \neq \emptyset, \mathbb{L}^{F}$ is "pseudo-axiomatic" in the sense of [35, p. 178]. It is easy to see that $\Lambda_{\mathbf{C}}(F)=\Lambda\left(\mathbf{C}^{F}\right)$, and also that $\Lambda(\mathbf{C})=\Lambda_{\mathbf{C}}(\mathbf{C}(\emptyset))$. It is interesting to note that the operators $\Lambda_{\mathbf{C}}$ and $\widetilde{\Omega}_{\mathfrak{A}}$ are both order-preserving (on subsets and on abstract logics, respectively), and satisfy

$$
\widetilde{\Omega}_{\mathfrak{A}}\left(\mathcal{C}^{F}\right) \subseteq \Omega_{\mathfrak{A}}(F) \quad \text { and } \quad \widetilde{\Omega}_{\mathfrak{A}}\left(\mathcal{C}^{F}\right) \subseteq \Lambda_{\mathbf{C}}(F) \quad \text { for any } F \in \mathcal{C} .
$$

Although the Frege operator is not strictly related to the algebraic structure, sometimes it will be more convenient to write $\Lambda(\mathbb{L})$ and $\Lambda_{\mathbb{L}}$ instead of $\Lambda(\mathbf{C})$ and $\Lambda_{\mathbf{C}}$; this will happen, in particular, when the abstract logic in question is a deductive system.

Actually, when applied to the original deductive system $\mathcal{S}=\langle\mathfrak{F m}, \vdash \mathcal{S}\rangle$, these concepts take a familiar form: $\Lambda(\mathcal{S})$ is the "interderivability relation of $\mathcal{S}$ " $\left(\varphi \vdash_{\mathcal{S}}\right.$ $\psi$ ), while for any $\Gamma \subseteq F m, \Lambda_{\mathcal{S}}(\Gamma)$ is the "interderivability relation of $\mathcal{S}$ with 
respect to the theory axiomatized by $\Gamma "\left(\Gamma, \varphi \vdash_{\mathcal{S}} \psi\right.$ and $\left.\Gamma, \psi \vdash_{\mathcal{S}} \varphi\right)$. On the other hand, it is easy to check that $\widetilde{\Omega}(\mathcal{S})$ is the congruence relation actually used in the most familiar cases to obtain the so-called "Tarski-Lindenbaum algebra" (e.g., the relation $\vdash \varphi \leftrightarrow \psi$ for classical, intuitionistic, and modal logic, etc.). In this case (5) takes a particularly simple form:

$$
\langle\varphi, \psi\rangle \in \widetilde{\Omega}(\mathcal{S}) \Leftrightarrow \gamma(\varphi) \vdash_{\mathcal{S}} \gamma(\psi) \text { for all } \gamma(p) \in F m .
$$

It is interesting to note how the concept of protoalgebraicity of a deductive system can be expressed in terms of the Frege operator and the (extended) Leibniz operator $\widetilde{\Omega}$; actually the original Definition 2.1 in [2] is

$$
\mathcal{S} \text { is protoalgebraic } \Leftrightarrow \forall \Gamma \in T h(\mathcal{S}), \Omega(\Gamma) \subseteq \Lambda_{\mathcal{S}}(\Gamma)
$$

and it is easy to check that the equivalent condition that $\Omega_{\mathfrak{A}}$ be order-preserving on $\mathcal{S}$-filters (the "correspondence property") can be expressed as

$$
\Leftrightarrow \forall \mathfrak{A}, \text { if } \mathcal{C} \subseteq \mathcal{F} i_{\mathfrak{A}}^{\mathcal{S}} \text { then } \widetilde{\Omega}_{\mathfrak{A}}(\mathcal{C})=\Omega_{\mathfrak{A}}(\mathbf{C}(\emptyset))
$$

and also that it is equivalent to have a particular case:

$$
\Leftrightarrow \forall \mathfrak{A}, \forall F \in \mathcal{F} i_{\mathfrak{A}}^{\mathcal{S}}, \widetilde{\Omega}_{\mathfrak{A}}\left(\left(\mathcal{F} i_{\mathfrak{A}}^{\mathcal{S}}\right)^{F}\right)=\Omega_{\mathfrak{A}}(F) .
$$

Since it is enough to have the correspondence property on $\mathfrak{F} \mathfrak{m}$, we can also write

$$
\Leftrightarrow \forall \Gamma \in T h(\mathcal{S}), \widetilde{\Omega}\left(\mathcal{S}^{\Gamma}\right)=\Omega(\Gamma) .
$$

We thus see that in the study of protoalgebraic logics, the extended Leibniz congruence of a family of filters reduces to the ordinary Leibniz congruence of the intersection of all filters in the family. This is the reason why our approach will be specially useful for non-protoalgebraic deductive systems. In particular, note that

$$
\text { If } \mathcal{S} \text { is protoalgebraic, then } \widetilde{\Omega}(\mathcal{S})=\Omega(\mathbf{C}(\emptyset)) .
$$

It is interesting to relate identity (10) with the result proved in [13] that a deductive system is protoalgebraic if and only if it satisfies a very general (namely, a parametrized and local) version of the Deduction Theorem. Both facts say, in different senses, that protoalgebraic deductive systems are, to some extent, determined by their theorems.

Two more classes of deductive systems can be defined in terms of the Frege operator:

Definition 1. $\mathcal{S}$ is selfextensional if and only if $\Lambda(\mathcal{S}) \in \operatorname{Con}(\mathfrak{F m})$, that is, if and only if $\Lambda(\mathcal{S})=\widetilde{\Omega}(\mathcal{S})$.

Selfextensional deductive systems have been studied mainly by Wójcicki, who has shown that they are those having an adequate class of referential matrices (see [56, Section 5.6]). A smaller class of deductive systems is the following:

Definition 2. $\mathcal{S}$ is Fregean if and only if $\forall \Gamma \in T h(\mathcal{S}), \mathcal{S}^{\Gamma}$ is selfextensional, that is, $\Lambda_{\mathcal{S}}(\Gamma) \in \operatorname{Con}(\mathfrak{F m})$, or, what amounts to the same, $\Lambda_{\mathcal{S}}(\Gamma)=\Lambda\left(\mathcal{S}^{\Gamma}\right)=\widetilde{\Omega}\left(\mathcal{S}^{\Gamma}\right)$. 
Since $\mathcal{S}=\mathcal{S}^{\mathrm{C}_{\mathcal{S}}(\emptyset)}$, every Fregean deductive system is also selfextensional. From (6) applied to $\mathcal{S}^{\Gamma}$ it follows that

- $\mathcal{S}$ is Fregean if and only if for any $\Gamma \in T h(\mathcal{S})$, and any $\varphi, \psi \in F m$, if $\Gamma, \varphi \dashv \vdash_{\mathcal{S}} \Gamma, \psi$ then for all $\gamma(p) \in F m$ we have $\Gamma, \gamma(\varphi) \vdash_{\mathcal{S}} \Gamma, \gamma(\psi)$,

and so we see that these deductive systems enjoy a very strong property of replacement of equivalents. Thus the name "Fregean" seems appropriate for such logics. Note that this term has been applied by Don Pigozzi in [36] to varieties of algebras; and in view of some of his results it seems that both uses are compatible.

The classification of deductive systems. So far we have two classifications of deductive systems: one according to the behaviour of the Leibniz operator, another according to the behaviour of the Frege operator. If we combine both we get a more complicated diagram, essentially shown in Figure 1. This diagram is very interesting, and thinking about it raises many interesting observations, QUESTIONS and PROBLEMS, some of them probably easy, but some maybe difficult.

By Theorem 2, which I will prove at the end of the talk, sectors number 4 and 7 are empty, and in sector 10 there are only the almost inconsistent deductive systems. I have not yet found any examples in sectors 6 and 9, but I think this will be easily solved. However, sector 2 is also empty as yet, and it would not surprise me if someone proved it is, although I do not dare to make any conjecture. I list below some deductive systems that I know have already been located into the diagram:

Sector 1: The inconsistent logics, $\mathbf{I P C}_{\rightarrow}$, and all its axiomatic extensions; this includes intuitionistic and classical logics and all intermediate (propositional) logics in the ordinary sense (i.e., axiomatic extensions of IPC).

Sector 3: All normal modal logics (understood as deductive systems extending $\mathbf{K}$ and having the strong necessitation rule $\varphi \vdash \square \varphi$ ) [4,34], relevance logics $\mathbf{R}$ and RM [22, 23], BCK-logic, all many-valued logics of Łukasiewicz [4, 46], the normal modal four-valued logic of [45], the systems $\mathcal{H}^{1}$ and $\mathcal{H}_{2}$ of $[18,30]$, Rasiowa's logic $J_{\nu}$ with semi-negation $[56,5.7 .2]$.

Sectors 5 and 8 include all quasi-normal modal logics (i.e., extensions of $\mathbf{K}$ but having only the weak rule of necessitation $\vdash \varphi \Rightarrow \vdash \square \varphi)$, [56, 34]; some of them are in sector 8, like $\mathbf{K}_{M P}$ and $\mathbf{T}_{M P}$, while others, like $\mathbf{S} \mathbf{4}_{M P}$ and $\mathbf{S} \mathbf{5}_{M P}$, are in sector 5, together with the quasi-normal four-valued modal logic studied in [21].

Sector 11: The deductive systems defined by sequent calculi $\mathcal{G}_{1}$ and $\mathcal{G}^{1}$ introduced in [30] and studied in [18].

Sector 12: The deductive system defined by a sequent calculus $\mathcal{G}_{0}$ introduced in $[30]$.

Sector 13: The fragments of classical logic having the following connectives: $\{\wedge\},\{\vee\}[32],\{\wedge, \vee\}[27]$; the deductive system $\mathbf{I P C}{ }^{*}$, i.e., the $\{\wedge, \vee, \neg\}$-fragment of intuitionistic logic $[4,44]$. 


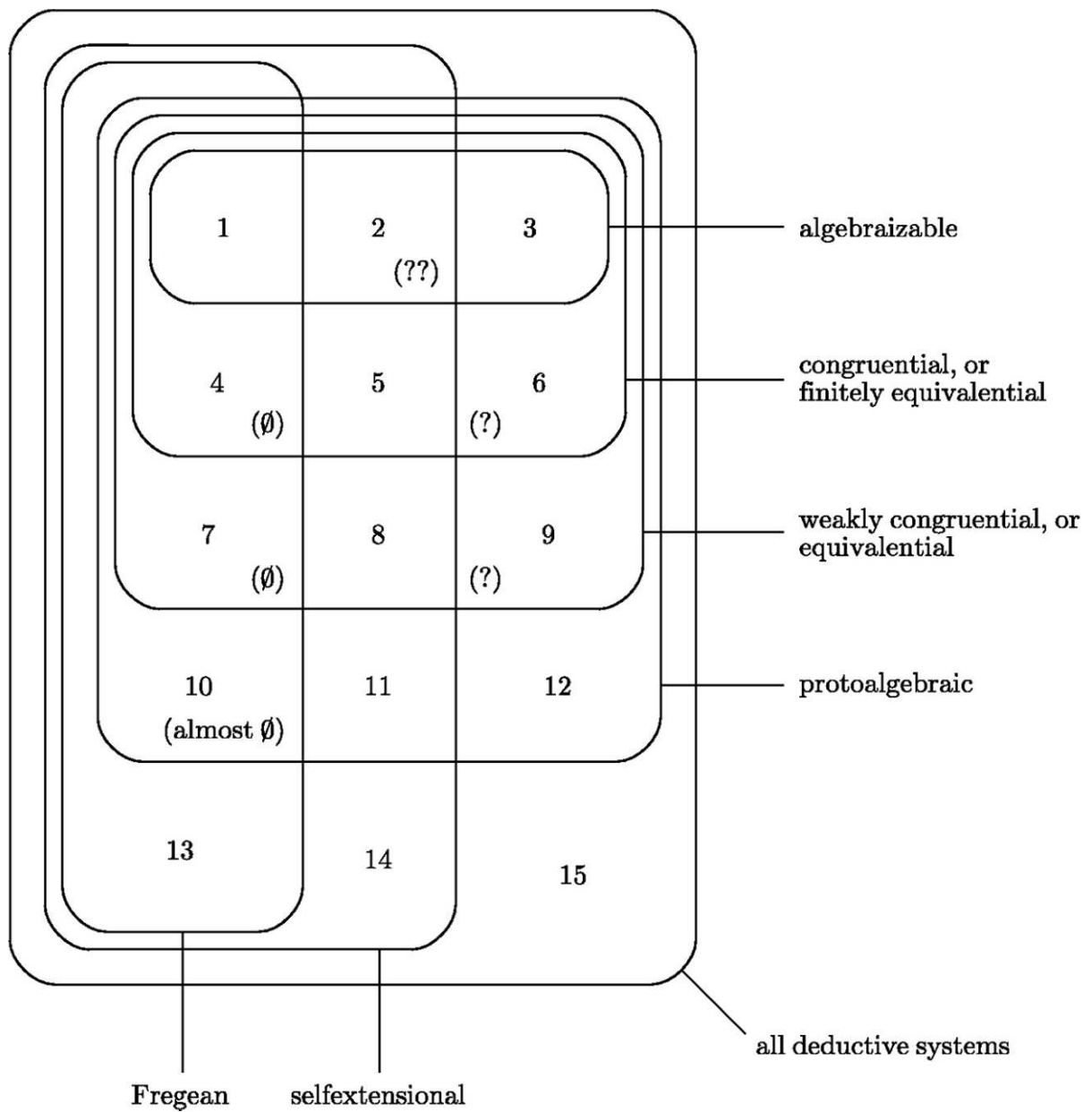

Fig. 1. The calssification of deductive systems

Sector 14: Belnap's four-valued logic [1], the weak version of $\mathbf{R}$ studied in [23], the logic of lattices analyzed in [44].

Sector 15, finally, contains at least all deductive systems obtained from a non-selfextensional one (with theorems) by adding to its theories the empty set.

It would be very interesting to find more "natural" examples in this last sector. In general, an interesting TASK is to classify every known propositional logic into this diagram. This would give us more insight into the algebraic properties of the deductive systems, and into the relative strength of the properties defining these classes of deductive systems.

Using abstract logics to characterize algebras. For protoalgebraic logics, the standard way to establish the relationship between the logic and a class of algebras is to use the algebras as matrices, and to determine the class of reduced 
matrices; in the extreme case of algebraizable logics this can be done by using the equational consequence of the class of algebras. Our early experience in Barcelona has made us think that there is another kind of relationship between logics and algebras, which also works for non-protoalgebraic logics, and which reduces to the old one for the protoalgebraic ones.

Roughly speaking, it consists in selecting some of the metalogical properties of the logic in a form such that the algebras in the class are characterized precisely by having a closure operator with these metalogical properties and which defines the identity in the algebra.

In some sense one can consider this approach as an extension to arbitrary algebras of Tarski's idea in [47] of an "axiomatic" presentation of the consequence operator of classical sentential logic (for more information see [3, 14]). Observe that in this early paper Tarski does not explicitly presuppose to be working on a (free) algebra of formulas, but his 1930-style description of sentences ("Aussage") as "inscriptions of completely determined structure" can be considered as equivalent to it, in the domain of sentential logics. Anyway, such "axiomatic" approach to the consequence operator of particular deductive systems was only recovered in [38], and later on in [31] and [39], where IPC and each of its usual fragments is characterized (using Kripke models) as the least deductive system whose closure operator satisfies certain metalogical properties (like PC, PDI, PDED, etc. quoted below). See also [8] and [56, Section 2.3].

While the mentioned papers and book focus on deductive systems, and thus deal only with closure operators on the algebra of formulas, the first papers I know focusing on the algebras and considering abstract logics on abstract algebras are [9] and [10], published jointly in a volume with an interesting preface by Roman Suszko. Actually, an early result of the kind we want is implicit in [9]: As an immediate corollary to its Theorem 3 one can prove that

$\mathfrak{A}$ is a Boolean algebra $\Leftrightarrow$ There is a closure operator $\mathbf{C}$ on $A$ such that:

- $\mathbf{C}$ is finitary and $\mathbb{L}=\langle\mathfrak{A}, \mathbf{C}\rangle$ is reduced;

- The PRA (Reductio ad Absurdum): $a \in \mathbf{C}(X) \Leftrightarrow \mathbf{C}(X, \neg a)=A$;

- The PDI (Disjunction): $\mathbf{C}(X, a \vee b)=\mathbf{C}(X, a) \cap \mathbf{C}(X, b)$;

and then $\mathcal{C}$ is the set of all filters of the Boolean algebra $\mathfrak{A}$.

This line of research has been followed by some of the people in Barcelona since the late seventies. Verdú ([50]-[54]) proved several theorems characterizing a class $\mathbb{K}$ of algebras by a set of conditions $\mathcal{P}$ on a closure operator in the following common form (now slightly reformulated):

$$
\begin{aligned}
\mathfrak{A} \in \mathbb{K} \Leftrightarrow \exists \mathbf{C} \text { on } A \text { such that } \mathbf{C} \text { is finitary, the abstract } \\
\qquad \text { logic } \mathbb{L}=\langle\mathfrak{A}, \mathbf{C}\rangle \text { is reduced, and satisfies properties } \mathcal{P} .
\end{aligned}
$$

Table 1 shows a sample list including the most well-known cases appearing in [52]. In it we find the familiar properties PDED (the Deduction Theorem) and 
PC (Conjunction) together with PPRA (Pseudo-Reductio ad Absurdum, or intuitionistic form of PRA) and weak versions of Disjunction (PWDI) and Reductio ad Absurdum (PWRA), as well other conditions.

Table 1. The " $\mathbb{K}-\mathcal{P}$ table"

\begin{tabular}{|c|c|}
\hline $\mathbb{K}$ & $\mathcal{P}$ \\
\hline lattice & $\begin{array}{c}\text { PC : } \mathbf{C}(a \wedge b)=\mathbf{C}(a, b)+ \\
\mathrm{PWDI}: \mathbf{C}(a \vee b)=\mathbf{C}(a) \cap \mathbf{C}(b)\end{array}$ \\
\hline distributive lattice & $\mathrm{PC}+\mathrm{PDI}$ \\
\hline Hilbert algebra & PDED $: a \rightarrow b \in \mathbf{C}(X) \Leftrightarrow b \in \mathbf{C}(X, a)$ \\
\hline implication algebra & $\begin{array}{c}\mathrm{PDED}+((a \rightarrow b) \rightarrow a) \rightarrow a \in \mathbf{C}(\emptyset) \\
\mathrm{PDED}+\mathcal{C} \text { has a basis of maximal sets }\end{array}$ \\
\hline Heyting algebra & $\begin{array}{c}\text { PDED }+\mathrm{PC}+\mathrm{PWDI}+ \\
\exists 0 \in A \text { such that } \mathbf{C}(0)=A \\
\mathrm{PDED}+\mathrm{PC}+\mathrm{PWDI}+ \\
\mathrm{PPRA}: \neg a \in \mathbf{C}(X) \Leftrightarrow \mathbf{C}(X, a)=A\end{array}$ \\
\hline Boolean algebra & $\begin{array}{c}\text { PDED + PWRA: } a \in \underset{\text { or }}{\mathbf{C}(b) \Leftrightarrow \mathbf{C}(b, \neg a)=A} \\
\text { PC }+ \text { PWRA }\end{array}$ \\
\hline
\end{tabular}

Later on, in [25, 34], similar results have been obtained for many classes of modal algebras related to normal and quasi-normal modal logics, either with a classical or an intuitionistic base. In the modal cases the characterizations are more involved, and one needs to combine several closure operators and several algebraic structures on the same set; this shows the usefulness of treating abstract logics as single mathematical objects. Also, similar works have been done for De Morgan algebras [24, 26] (related to Belnap's four-valued logic [1]) and several of its subclasses related to relevance logic $\mathbf{R}$ [23], to a modal extension of Belnap's logic [21, 45], and to Eukasiewicz's infinite-valued logic [49]. A weakened version of PDED, where $|X| \leq 1$, was already considered in [37], where the algebras characterized by this and Modus Ponens were found, and called "quasi-Hilbert algebras"; this topic has been studied in detail in $[18,30]$. Some other cases are under study.

A first reading of theorems (11) could be purely algebraic: The identity in algebras in $\mathbb{K}$ is characterized by the closure system $\mathcal{C}$ of all (lattice-, implicative-, ...) filters on the algebra, and this closure system or its associated closure operator $\mathbf{C}$ is characterized by properties $\mathcal{P}$. Indeed, Verdú found that for all cases appearing in Table 1, and also for others, a strong link between closure operators and congruences could be established, in the following common form:

(12) For any algebra $\mathfrak{A}$ there is an isomorphism between the lattice of all finitary closure operators on $A$ which satisfy the properties $\mathcal{P}$ and the lattice $\mathrm{Con}_{\mathbb{K}}(\mathfrak{A})$

(many in [53], unpublished; see also [17, Theorem 4], [25, comment on p. 1048] and [27, Theorem 4.7]; in each of the particular cases treated, these sets were proved 
to be lattices, and we will see that there is a general reason for this fact). This apparently strengthens the purely algebraic reading. There is, however, another reading, since in almost all cases appearing in Table 1 properties $\mathcal{P}$ characterize a deductive system, as proved in $[31,38,39,56]$; in general, each of the classes $\mathbb{K}$ under consideration bears some relationship with a deductive system, but while for the classes $\mathbb{K}$ associated with an algebraizable, or at least protoalgebraic, logic the connection is given by the standard method, the link is not so clear in the remaining cases. To better understand our approach it may be useful to review more closely the case of distributive lattices, treated in detail in [27], [28] and [19].

A case study: $\mathrm{CPC}_{\wedge, \vee}$ and distributive lattices. Now $\mathfrak{F m}=\langle F m, \wedge, \vee\rangle$ is of type $(2,2)$; by $\mathbb{D}$ we denote the variety of distributive lattices, which is generated by $\underline{\mathbf{2}}=\langle 2, \wedge, \vee\rangle$ where $2=\{0,1\}$ with $0<1$. We call $\mathcal{S}_{2}$ the deductive system generated by the matrix $\langle\underline{\mathbf{2}},\{1\}\rangle$ in the usual way. It is obvious that $\mathcal{S}_{2}$ $=\mathrm{CPC}_{\wedge, \vee}$, the $\{\wedge, \vee\}$-fragment of CPC (= Classical Propositional Calculus); a particularly useful axiomatization of $\mathcal{S}_{2}$ by a set of twelve Hilbert-style rules (and no axioms, since this logic has no theorems) appears in [16]. The following facts concerning this logic and this variety have been proved:

- $\mathbb{D}$ is not the equivalent algebraic semantics for any algebraizable logic $[27,2.1]$.

- $\mathcal{S}_{2}$ is not protoalgebraic $[27,2.8]$, but it is Fregean (implicit in [27, 4.11]).

- The class of algebras corresponding to the reduced matrices of $\mathcal{S}_{2}$ is not, as one might hope [55, page 162], the class $\mathbb{D}$; actually:

- $\operatorname{Alg}^{*}\left(\mathcal{S}_{2}\right)=\{\mathfrak{A} \in \mathbb{D}: \exists 1=\max \mathfrak{A}$, and $\forall a, b \in A, a<b \Rightarrow \exists c \in A$ with $a \vee c \neq 1$ and $b \vee c=1\}$ [19]; this class is not even a quasivariety.

But we can look in another direction. Two facts follow immediately from the definitions:

- $\varphi \dashv \vdash_{\mathcal{S}_{2}} \psi \Leftrightarrow \vDash_{\mathbb{D}} \varphi \approx \psi$ for any $\varphi, \psi \in F m$.

- $\left\{\varphi_{1}, \ldots, \varphi_{n}\right\} \vdash_{\mathcal{S}_{2}} \psi \Leftrightarrow$ the inequality $\varphi_{1} \wedge \ldots \wedge \varphi_{n} \preccurlyeq \psi$ is "satisfied" in $\mathbb{D} \Leftrightarrow \psi^{\mathfrak{A}}(h) \in \boldsymbol{F i l t}\left[\varphi_{1}^{\mathfrak{A}}(h), \ldots, \varphi_{n}^{\mathfrak{A}}(h)\right]$ for any interpretation $h$ in any $\mathfrak{A} \in \mathbb{D}$, where the operator Filt means "filter-generation" in the lattice $\mathfrak{A}$.

So it seems that logical inference in $\mathcal{S}_{2}$ "corresponds" to filter-generation in $\mathbb{D}$. In order to make this correspondence more precise we have used the notion of model of a Gentzen calculus, developed from a suggestion made by Wim Blok.

Definition 3. In general, if (risking some confusion) we denote a sequent by $\Gamma \vdash \varphi$, where $\Gamma$ is a finite set of formulas, and $\varphi$ is a formula, then we say that

$$
\mathbb{L}=\langle\mathfrak{A}, \mathbf{C}\rangle \text { is a model of the Gentzen style rule } \frac{\left\{\Gamma_{i} \vdash \varphi_{i}: i \in I\right\}}{\Gamma \vdash \varphi}
$$

if and only if for all interpretations $h$ in $\mathfrak{A}$,

$$
\text { if } \forall i \in I, \varphi_{i}^{\mathfrak{A}}(h) \in \mathbf{C}\left(\Gamma_{i}^{\mathfrak{A}}(h)\right) \text { then } \varphi^{\mathfrak{A}}(h) \in \mathbf{C}\left(\Gamma^{\mathfrak{A}}(h)\right) \text {. }
$$


In the case of $\mathcal{S}_{2}$, one sequent calculus for it consists of the ordinary structural rules and the ordinary rules for conjunction and disjunction:

$$
\begin{array}{cl}
\varphi \vdash \varphi \quad \frac{\Gamma \vdash \varphi}{\Gamma, \psi \vdash \varphi} & \frac{\Gamma \vdash \varphi \Gamma, \varphi \vdash \psi}{\Gamma \vdash \psi} \\
\frac{\Gamma, \varphi, \psi \vdash \xi}{\Gamma, \varphi \wedge \psi \vdash \xi} & \frac{\Gamma \vdash \varphi \quad \Gamma \vdash \psi}{\Gamma \vdash \varphi \wedge \psi} \\
\frac{\Gamma, \varphi \vdash \xi \quad \Gamma, \psi \vdash \xi}{\Gamma, \varphi \vee \psi \vdash \xi} & \frac{\Gamma \vdash \varphi}{\Gamma \vdash \varphi \vee \psi} \quad \frac{\Gamma \vdash \psi}{\Gamma \vdash \varphi \vee \psi}
\end{array}
$$

Obviously any abstract logic is a model of the structural rules. And it is easy to see that an abstract logic $\mathbb{L}$ is a model of this calculus if and only if it satisfies PC and PDI. From this wide class of models we select the so-to-speak "regular" ones:

$\mathbb{L}$ is distributive $\Leftrightarrow \mathbf{C}$ is finitary, satisfies $\mathrm{PC}, \mathrm{PDI}$, and $\mathbf{C}(\emptyset)=\emptyset$.

For any $\mathfrak{A}$ we denote by $\mathbb{D}(\mathfrak{A})$ the set of all distributive abstract logics on $\mathfrak{A}$.

This definition is slightly different from the one actually used in [27], but I need it here in this way. In [27] distributive abstract logics are studied from several points of view, in particular as logics projectively generated from $\langle\underline{\mathbf{2}},\{1\}\rangle$ by sets of homomorphisms. Some of the results of [27] are relevant here (if $\mathfrak{A} \in \mathbb{D}$ then I denote by $\mathcal{F} i l t(\mathfrak{A})$ the closure system of all lattice filters of $\mathfrak{A}$ plus the empty set):

- For any $\mathfrak{A}$, the least abstract distributive logic on $\mathfrak{A}$ is $\left\langle\mathfrak{A}, \mathcal{F} i_{\mathfrak{A}}^{\mathcal{S}_{2}}\right\rangle$ [Theorem 3.11.2].

- $\mathbb{L} \in \mathbb{D}(\mathfrak{A})$ if and only if there is a bilogical morphism from $\mathbb{L}$ onto an abstract $\operatorname{logic} \mathbb{L}^{\prime}=\left\langle\mathfrak{A}^{\prime}, \mathbf{C}^{\prime}\right\rangle$ such that $\mathfrak{A}^{\prime} \in \mathbb{D}$ and $\mathcal{C}^{\prime}=\mathcal{F}$ ilt $\left(\mathfrak{A}^{\prime}\right)$ [Theorem 4.2].

- $\mathbb{L}=\langle\mathfrak{A}, \mathbf{C}\rangle$ is a reduced distributive abstract logic if and only if $\mathfrak{A} \in \mathbb{D}$ and $\mathcal{C}=\mathcal{F}$ ilt $(\mathfrak{A})$ [Corollary 4.4].

- An algebra (of type $(2,2)$, of course) is a distributive lattice if and only if it is the algebra reduct of a reduced distributive abstract logic [Corollary 4.5].

- If $\mathbb{L} \in \mathbb{D}(\mathfrak{A})$ then $\widetilde{\Omega}_{\mathfrak{A}}(\mathbb{L})=\Lambda(\mathbb{L})$, and this mapping gives a lattice isomorphism between $\mathbb{D}(\mathfrak{A})$ and $\mathrm{Con}_{\mathbb{D}}(\mathfrak{A})$ [Theorem 4.7].

As a particular case we conclude that if $\mathfrak{A} \in \mathbb{D}$ then $\operatorname{Con}(\mathfrak{A}) \cong \mathbb{D}(\mathfrak{A})$, an interesting representation result for the congruence lattice of a distributive lattice. Going back to our deductive system $\mathcal{S}_{2}$, by taking $\mathfrak{A}=\mathfrak{F} \mathfrak{m}$ we obtain:

- The least distributive abstract logic on $\mathfrak{F m}$ is $\mathcal{S}_{2}$ [Theorem 3.9].

- There is no other consistent distributive deductive system than $\mathcal{S}_{2}$ [Corollary 4.8].

- $\widetilde{\Omega}: \mathbb{D}(\mathfrak{F} \mathfrak{m}) \cong T h\left(\vDash_{\mathbb{D}}\right)$ [Corollary 4.10].

- For every $\Gamma \in T h\left(\mathcal{S}_{2}\right), \mathcal{S}_{2}^{\Gamma}$ is distributive, and the mapping $\Gamma \mapsto \widetilde{\Omega}\left(\mathcal{S}_{2}^{\Gamma}\right)=$ $\Lambda\left(\mathcal{S}_{2}^{\Gamma}\right)$ gives an embedding of $T h\left(\mathcal{S}_{2}\right)$ into $T h\left(\vDash_{\mathbb{D}}\right)$ [Proposition 4.12]. 
So we see there are new and strong links between $\mathcal{S}_{2}$ and $\mathbb{D}$, going beyond the completeness theorem, and similar to some extent to those found for algebraizable logics. These links, however, have been obtained by using the notion of distributive logic, which arises here as a notion of model of a particular Gentzen calculus for $\mathcal{S}_{2}$. In general, it is easy to see that this notion of model is not an invariant of the deductive system defined by the calculus, but depends on the calculus itself; actually, if we take the rules of any Hilbert-style presentation of a deductive system and use them as "axioms" for a Gentzen-style calculus having also the structural rules, then it is easy to see that the Gentzen calculus so obtained has among its models all (!) abstract logics whose closed sets are filters of the deductive system. This notion has proved to be particularly fruitful in the algebraic study of Gentzen systems (see $[18,20,32,43,48]$ ). But if one wants to focus on the study of deductive systems, then this dependence should be removed. In other words, there should be a characterization of the class $\mathbb{D}(\mathfrak{A})$ by its relationship with $\mathcal{S}_{2}$ without making any reference to a particular form of definition of the deductive system.

Outline of a general theory. To end this talk I will present one proposal for building a general theory to achieve this desire, and such that several of the links outlined above between $\mathcal{S}_{2}$ and $\mathbb{D}$, and other theorems like (11) and (12), become particular cases of general properties. This proposal originated in Ramon Jansana's proof of Theorem 1, and will be dealt with in detail in [20]; here I will only comment on the main definitions and results, and apply them to prove Theorem 2 as announced before.

Definition 4. Let $\mathcal{S}$ be any deductive system. An algebra $\mathfrak{A}$ is an $\mathcal{S}$-algebra if and only if the abstract logic $\left\langle\mathfrak{A}, \mathcal{F} i_{\mathfrak{A}}^{\mathcal{S}}\right\rangle$ is reduced, i.e., if and only if $\widetilde{\Omega}_{\mathfrak{A}}\left(\mathcal{F} i_{\mathfrak{A}}^{\mathcal{S}}\right)=$ $\Delta_{A}$. The class of all $\mathcal{S}$-algebras will be denoted by $\operatorname{Alg}(\mathcal{S})$.

The following facts can be proved:

- $A l g(\mathcal{S}) \supseteq A l g^{*}(\mathcal{S})$, but both classes of algebras generate the same variety; actually, this is the variety generated by $\mathfrak{F m} / \widetilde{\Omega}(\mathcal{S})$, the "Tarski-Lindenbaum algebra of $\mathcal{S}$ ". (PROBLEM: Do they generate the same quasivariety?)

- If $\mathcal{S}$ is protoalgebraic then $\operatorname{Alg}(\mathcal{S})=\operatorname{Alg}^{*}(\mathcal{S})$. The converse is false; examples are all logics mentioned before as belonging to sector 15 of Figure 1. (PROBLEM: Are there any other examples?)

- If $\mathcal{S}$ is algebraizable then $\operatorname{Alg}(\mathcal{S})$ is the equivalent algebraic semantics for $\mathcal{S}$.

The PROBLEM of definability of $\operatorname{Alg}(\mathcal{S})$ must be a hard one, since it is so already for $\operatorname{Alg}^{*}(\mathcal{S})$, which can be dealt with in a first-order language; while for an algebraizable $\mathcal{S}$ we know that it is a quasivariety, we have seen that $\operatorname{Alg}^{*}\left(\mathcal{S}_{2}\right)$ is not (see [42] for a deeper analysis, including an example where it is not even elementary). The treatment of similar questions for $\operatorname{Alg}(\mathcal{S})$ needs, in principle, a second-order language. 
The term "S -algebra" is taken from [40], where it is used to refer to $\operatorname{Alg}^{*}(\mathcal{S})$ in the very restricted case of "implicational logics" (see also [56, Section 3.5]). Since these logics are algebraizable, and thus protoalgebraic, we see that our choice is coherent with these early uses. However, to say that this class of algebras is a true generalization of the usual algebraic semantics for protoalgebraic logics we need a way to express them as the algebra reducts of some kind of "reduced models" for $\mathcal{S}$; the notion of "model" we propose is the following:

Definition 5. Let $\mathcal{S}$ be any deductive system. An abstract logic $\mathbb{L}=\langle\mathfrak{A}, \mathcal{C}\rangle$ is an $\mathcal{S}$-logic if and only if $\mathcal{C} / \widetilde{\Omega}_{\mathfrak{A}}(\mathbb{L})=\mathcal{F} i_{\mathfrak{A} / \widetilde{\Omega}_{\mathfrak{A}}(\mathbb{L})}$. The class of all $\mathcal{S}$-logics will be denoted by $\log (\mathcal{S})$, and the class of all $\mathcal{S}$-logics on an algebra $\mathfrak{A}$ by $\log _{\mathcal{S}}(\mathfrak{A})$.

Again the following facts can be proved:

- If $\mathbb{L} \in \log _{\mathcal{S}}(\mathfrak{A})$ then $\mathfrak{A} / \widetilde{\Omega}_{\mathfrak{A}}(\mathbb{L}) \in \operatorname{Alg}(\mathcal{S})$.

- The reduced $\mathcal{S}$-logics are $\left\langle\mathfrak{A}, \mathcal{F} i_{\mathfrak{A}}^{\mathcal{S}}\right\rangle$ for $\mathfrak{A} \in A l g(\mathcal{S})$. That is, the $\mathcal{S}$-algebras are the algebraic reducts of the reduced $\mathcal{S}$-logics, and in this sense the situation is similar to the relationship between $\operatorname{Alg}^{*}(\mathcal{S})$ and $\operatorname{Matr}(\mathcal{S})$.

- If $\mathbb{L} \in \log _{\mathcal{S}}(\mathfrak{A})$ then $\mathcal{C} \subseteq \mathcal{F} i_{\mathfrak{A}}^{\mathcal{S}}$, and as a consequence:

- The least $\mathcal{S}$-logic on $\mathfrak{A}$ is $\left\langle\mathfrak{A}, \mathcal{F}_{\mathfrak{A}}^{\mathcal{S}}\right\rangle$, and also:

- The least $\mathcal{S}$-logic on $\mathfrak{F m}$ is $\mathcal{S}$ itself.

So $\mathcal{S}$-logics are families of $\mathcal{S}$-filters (that is, "generalized matrices" in the sense of [56]) which in some sense are "saturated": they give all $\mathcal{S}$-filters in the quotient algebra. One can also prove the following

Proposition 1 (Completeness). For any $\Gamma \subseteq F m$ and any $\varphi \in F m, \Gamma \vdash \mathcal{S} \varphi$ if and only if $\varphi^{\mathfrak{A}}(h) \in \mathbf{F i}_{\mathfrak{A}}^{\mathcal{S}}\left(\Gamma^{\mathfrak{A}}(h)\right)$ for every interpretation $h$ in any $\mathfrak{A} \in A l g(\mathcal{S})$; here $\mathbf{F i}_{\mathfrak{A}}^{\mathcal{S}}$ is the closure operator associated with the closure system $\mathcal{F} i_{\mathfrak{A}}^{\mathcal{S}}$.

The interest of this completeness result depends on how nice characterizations of the class $\operatorname{Alg}(\mathcal{S})$ and of the operator $\mathbf{F i}_{\mathfrak{A}}^{\mathcal{S}}$ on that class we have. And it may happen that we have these while having no, or not good, characterizations of the class of reduced matrices, which is the one commonly used for completeness; we can even have nice characterizations of algebras in $\operatorname{Alg}^{*}(\mathcal{S})$, but not of the $\mathcal{S}$-filters on them corresponding to the reduced matrices (see for instance [18]). In the case of $\mathcal{S}_{2}$ dealt with before, using some of the results from [27] quoted before, one can prove that $\operatorname{Alg}\left(\mathcal{S}_{2}\right)=\mathbb{D}$ and that $\log _{\mathcal{S}_{2}}(\mathfrak{A})=\mathbb{D}(\mathfrak{A})$ for every $\mathfrak{A}$ (note that this last fact would not be true with the notion of distributive abstract logic used in [27]).

Then a kind of PROGRAM could be formulated: Find and characterize in a nice way the classes $\operatorname{Alg}(\mathcal{S})$ and $\log (\mathcal{S})$ for all deductive systems $\mathcal{S}$ known in the literature. By "nice" I mean independent, purely algebraic definitions of $\operatorname{Alg}(\mathcal{S})$, and characterizations of $\log (\mathcal{S})$ using metalogical properties of $\mathcal{S}$, especially characterizations as the models of some Gentzen 
calculus for $\mathcal{S}$ (with additional "regularity" conditions such as being finitary, etc.). This in turn would give us a criterion to discriminate among different sequent calculi defining the same deductive system $\mathcal{S}$.

This program is interesting even for algebraizable logics, where $\operatorname{Alg}(\mathcal{S})$ is just the equivalent quasivariety semantics: the class $\log (\mathcal{S})$ still has to be nicely characterized in several cases. Note that the determination and nice characterization of $\log (\mathcal{S})$ as required before presupposes a sound knowledge of $\mathcal{S}$, both in its Hilbert-style aspect (the axioms, rules, theorems, ...) necessary to deal with $\mathcal{F} i_{\mathfrak{A}}^{\mathcal{S}}$, and in its metalogical properties, necessary to characterize $\log (\mathcal{S})$ as the models of some Gentzen calculus. Our experience shows that selfextensional deductive systems often admit such nice characterizations, while for non-selfextensional deductive systems they are usually indirect, for instance as abstract logics having some specific relationship with those in $\log \left(\mathcal{S}^{\prime}\right)$ where $\mathcal{S}^{\prime}$ is a selfextensional deductive system closely related to $\mathcal{S}$, often its "weak version" (i.e., $\mathcal{S}^{\prime}$ has the same axioms and inference rules as $\mathcal{S}$, except that some of the rules are restricted to theorems); this is the case, for instance, of normal modal logics [25, 34], of relevance system $\mathbf{R}$ [23], and of systems $\mathcal{H}^{1}$ and $\mathcal{H}_{2}$ of [18] (their weak versions being systems $\mathcal{G}^{1}$ and $\mathcal{G}_{1}$ respectively; see before). In many cases, however, it seems impossible to find a sequent calculus whose "regular" models are exactly all $\mathcal{S}$-logics (see for instance [33]).

In all cases mentioned in Table 1, it has been proved that the logics satisfying $\mathcal{P}$ are the $\mathcal{S}$-logics for the deductive system $\mathcal{S}$ usually associated with the class $\mathbb{K}$, which turns out to be the class of $\mathcal{S}$-algebras. If $\mathcal{S}$ has some proper name then this name is also applied to the $\mathcal{S}$-logics; thus we get a new and a posteriori justification of terms like "classical abstract logics" (already used in [9]), "intuitionistic (abstract) logics" [25], etc. In other cases, the $\mathcal{S}$-logics have inherited their proper name from the class of $\mathcal{S}$-algebras; this is, for instance, the origin of terms like "distributive logics" $[27,51]$ or "De Morgan logics" [24, 26]. And one can find quite often in the literature that a similar procedure has been followed to christen as "S $\mathcal{S}$-algebras", for a particular $\mathcal{S}$, a class of algebras related to $\mathcal{S}$, even when the relationship was not completely clarified, or simply when no standard term was available.

In general, the two classes $\operatorname{Alg}(\mathcal{S})$ and $\log (\mathcal{S})$ are linked by several properties not immediately contained in the definitions, and which will be proved in [20] using some tools of the theory of abstract logics.

Proposition 2. The algebraic category associated with $\operatorname{Alg}(\mathcal{S})$ (i.e., algebras and homomorphisms) is isomorphic to a full subcategory of the abstract-logical category associated with $\log (\mathcal{S})$ (i.e., abstract logics with logical morphisms, see $[10])$; the subcategory is that of reduced $\mathcal{S}$-logics. Moreover, if we restrict all arrows to be epimorphisms, then this full subcategory is also reflective, and the reflector is the functor associated with the factorization of $\mathbb{L}$ by $\widetilde{\Omega}_{\mathfrak{A}}(\mathbb{L})$.

Proposition 3. $\operatorname{Con}_{A l g(\mathcal{S})}(\mathfrak{A})$ is always a complete lattice, with inf $=\bigcap$. 
Thus after Theorem 1 also $\log _{\mathcal{S}}(\mathfrak{A})$ will become a complete lattice, isomorphic to the former:

Theorem 1 (Jansana). For any deductive system $\mathcal{S}$ and any algebra $\mathfrak{A}$, the (extended) Leibniz operator $\widetilde{\Omega}_{\mathfrak{A}}$ is an isomorphism between the complete lattices $\log _{\mathcal{S}}(\mathfrak{A})$ and $\operatorname{Con}_{A l g(\mathcal{S})}(\mathfrak{A})$.

Now it is clear that Theorem 4.7 of [27], quoted above, is just the particular case of this general result for $\mathcal{S}=\mathcal{S}_{2}$. The interest of this theorem lies both in its generality (it holds for any deductive system!) and in its applications. We hope that all theorems having the form (12) will also become particular cases of this result, after having conveniently characterized the classes of algebras and of closure operators there involved. On the other hand, from it and taking Theorem 5.1 of [4] into account, we can prove

Proposition 4. If $\mathcal{S}$ is algebraizable then, for any $\mathfrak{A}$, the lattice $\mathcal{F} i_{\mathfrak{A}}^{\mathcal{S}}$ is isomorphic to the lattice $\log _{\mathcal{S}}(\mathfrak{A})$ through the mapping $F \mapsto\left(\mathcal{F} i_{\mathfrak{A}}^{\mathcal{S}}\right)^{F}$.

This confirms one of the chief features of algebraizable logics we had already observed, that their $\mathcal{S}$-logics are all of the form "all $\mathcal{S}$-filters containing a given one", and thus their study can be reduced to the study of the $\mathcal{S}$-filters. And it turns out that the key point in our proof of Theorem 2 is a particular case of this property. We can first prove a weaker thing under different hypotheses:

Proposition 5. If $\mathcal{S}$ is, Fregean and $\mathbf{C}_{\mathcal{S}}(\emptyset) \neq \emptyset$ then the mapping $\Gamma \mapsto \mathcal{S}^{\Gamma}$ is an order-preserving embedding of $T h(\mathcal{S})$ into $\log _{\mathcal{S}}(\mathfrak{F m})$.

Then, using this and Theorem 1, we prove

Proposition 6. If $\mathcal{S}$ is protoalgebraic, Fregean, and satisfies $\mathbf{C}_{\mathcal{S}}(\emptyset) \neq \emptyset$, then the mapping $\Gamma \mapsto \mathcal{S}^{\Gamma}$ is an isomorphism between the lattices $T h(\mathcal{S})$ and $\log _{\mathcal{S}}(\mathfrak{F m})$.

On the other hand, we have the following general characterization of the finitarity of closure operators:

Proposition 7. A closure operator $\mathbf{C}$ on a set $A$ is finitary if and only if the operator $\Lambda_{\mathbf{C}}$ preserves unions of directed families of subsets of $A$.

In view of the above results the proof of the announced theorem is simple but may have some interest:

THEOREM 2. If a deductive system $\mathcal{S}$ is protoalgebraic and Fregean, and satisfies $\mathbf{C}_{\mathcal{S}}(\emptyset) \neq \emptyset$, then it is algebraizable.

Proof. If $\mathcal{S}$ is protoalgebraic then by $(9), \Omega(\Gamma)=\widetilde{\Omega}\left(\mathcal{S}^{\Gamma}\right)$ for any $\Gamma \in T h(\mathcal{S})$, thus the result of the composition of the isomorphisms of Theorem 1 and of Proposition 6 is just $\Omega: T h(\mathcal{S}) \cong \operatorname{Con}_{A l g(\mathcal{S})}(\mathfrak{F m})$, so $\Omega$ is injective and orderpreserving on $T h(\mathcal{S})$. On the other hand, $\mathcal{S}$ is both protoalgebraic and Fregean, 
therefore $\Omega=\Lambda_{\mathcal{S}}$ on $T h(\mathcal{S})$, and since by definition $\mathcal{S}$ is finitary, by Proposition 7 we conclude that $\Omega$ preserves unions of directed families of $T h(\mathcal{S})$. By Theorem 4.2 of [4] quoted before, it follows that $\mathcal{S}$ is algebraizable.

\section{References}

[1] N. D. Belnap, Jr., A useful four-valued logic, in: Modern Uses of Multiple-Valued Logic, J. M. Dunn and G. Epstein (eds.), Reidel, Dordrecht 1977, 8-37.

[2] W. J. Blok and D. Pigozzi, Protoalgebraic logics, Studia Logica 45 (1986), 337-369.

[3] -, - , Alfred Tarski's work on general metamathematics, J. Symbolic Logic 53 (1988), $36-50$.

[4] —, 一, Algebraizable logics, Mem. Amer. Math. Soc. 396 (1989).

[5] - - - Local deduction theorems in algebraic logic, in: Algebraic Logic, H. Andréka, J. D. Monk and I. Németi (eds.), Colloq. Math. Soc. János Bolyai 54, North-Holland, Amsterdam 1991, 75-109.

[6] - - - , The deduction theorem in algebraic logic, preprint, 1991, to appear.

[7] - - - Algebraic semantics for universal Horn logic without equality, in: Universal Algebra and Quasigroups, A. Romanowska and J. D. H. Smith (eds.), Heldermann, Berlin 1992, to appear.

[8] S. L. Bloom, A note on $\Psi$-consequences, Rep. Math. Logic 8 (1977), 3-9.

[9] S. L. Bloom and D. J. Brown, Classical abstract logics, Dissertationes Math. 102 (1973), $43-51$.

[10] D. J. Brown and R. Suszko, Abstract logics, ibid., 9-42.

[11] S. Burris and H. P. Sankappanavar, A Course in Universal Algebra, Springer, New York 1981.

[12] J. Czelakowski, Equivalential logics, I, II, Studia Logica 40 (1981), 227-236 and 355372 .

[13] J. Czelakowski and W. Dziobiak, A deduction theorem schema for deductive systems of propositional logics, Studia Logica, Special Issue on Algebraic Logic, 50 (1991), 385-390.

[14] J. Czelakowski and G. Malinowski, Key notions of Tarski's methodology of deductive systems, Studia Logica 44 (1985), 321-351.

[15] B. A. Davey and H. A. Priestley, Introduction to Lattices and Order, Cambridge Univ. Press, Cambridge 1990.

[16] K. Dyrda and T. Prucnal, On finitely based consequence determined by a distributive lattice, Bull. Sec. Logic Polish Acad. Sci. 9 (1980), 60-66.

[17] J. M. Font, On some congruence lattices of a topological Heyting lattice, in: Contributions to General Algebra 5, J. Czermak et al. (eds.), Teubner, Stuttgart 1987, 129-137.

[18] J. M. Font and J. L. García Lapresta, Logics and Algebras motivated by cardinality restrictions in the Deduction Theorem, manuscript, 1992.

[19] J. M. Font, F. Guzmán and V. Verdú, Characterization of the reduced matrices for the $\{\wedge, \vee\}$-fragment of classical logic, Bull. Sec. Logic Polish Acad. Sci. 20 (1991), 124-128.

[20] J. M. Font and R. Jansana, A general algebraic semantics for deductive systems, preprint, 1992, to appear.

[21] J. M. Font and M. Rius, A four-valued modal logic arising from Monteiro's last algebras, in: Proc. 20th Internat. Sympos. on Multiple-Valued Logic, Charlotte 1990, 85-92.

[22] J. M. Font and G. Rodríguez, Note on algebraic models for relevance logic, Z. Math. Logik Grundlag. Math. 36 (1990), 535-540.

[23] -, - Algebraic study of system $\mathbf{R}$ of relevance logic, manuscript, 1992. 
[24] J. M. Font and V. Verdú, Abstract characterization of a four-valued logic, in: Proc. 18th Internat. Sympos. on Multiple-Valued Logic, Palma de Mallorca 1988, 389-396.

[25] —, 一, A first approach to abstract modal logics, J. Symbolic Logic 54 (1989), 1042-1062.

[26] —, - Completeness theorems for a four-valued logic related to De Morgan lattices, Fac. Math. Preprint Ser. 57, Barcelona 1989.

[27] —, 一, Algebraic logic for classical conjunction and disjunction, Studia Logica, Special Issue on Algebraic Logic, 50 (1991), 391-419.

[28] - , - The lattice of distributive closure operators over an algebra, Studia Logica, to appear.

[29] - - - Algebraic study of Belnap's four-valued logic, manuscript.

[30] J. L. García Lapresta, Finitely deductive logics, Ph.D. dissertation, Univ. of Barcelona, 1991 (in Spanish).

[31] A. Grzegorczyk, An approach to logical calculi, Studia Logica 30 (1972), 33-43

[32] F. Guzmán and V. Verdú, On two Gentzen logifications of the variety of semilattices, manuscript, 1992.

[33] R. Jansana, The Box fragments of modal logic K, in: Actas del VII Congreso de Lenguajes Naturales y Lenguajes Formales, Vic, Barcelona 1991, C. Martín-Vide (ed.), 409-413 (in Spanish).

[34] —, Abstract modal logics, preprint, 1992, to appear.

[35] J. Łoś and R. Suszko, Remarks on sentential logics, Indag. Math. 20 (1958), 177-183.

[36] D. Pigozzi, Fregean algebraic logic, in: Algebraic Logic, H. Andréka, J. D. Monk and I. Németi (eds.), Colloq. Math. Soc. János Bolyai 54, North-Holland, Amsterdam 1991, 473-502.

[37] J. Pla and V. Verdú, Quasi-Hilbert algebras, Publ. Mat. 20 (1980), 97-99 (in Catalan).

[38] W. A. Pogorzelski and J. Słupecki, Basic properties of deductive systems based on nonclassical logics, I, II, Studia Logica 9 (1960), 163-176 and 10 (1960), 77-95.

[39] M. Porębska and A. Wroński, A characterization of fragments of the intuitionistic propositional logic, Rep. Math. Logic 4 (1975), 39-42.

[40] H. Rasiowa, An Algebraic Approach to Non-Classical Logics, North-Holland, Amsterdam 1974 .

[41] W. Rautenberg, Axiomatizing logics closely related to varieties, Studia Logica, Special Issue on Algebraic Logic, 50 (1991), 607-620.

[42] -, On reduced matrices, preprint, 1992, to appear.

[43] J. Rebagliato and V. Verdú, On the algebraization of some Gentzen systems, Fund. Inform., Special Issue on Algebra and Logic in Computer Science (1992), to appear.

[44] -, 一, A Hilbert-style axiomatization of the $\{\wedge, \vee, \neg\}$-fragment of IPC, manuscript, 1992.

[45] M. Rius, Tetravalent modal logics, Ph.D. dissertation, Univ. of Barcelona, 1992 (in Catalan).

[46] A. J. Rodríguez, A. Torrens and V. Verdú, Eukasiewicz logic and Wajsberg algebras, Bull. Sec. Logic Polish Acad. Sci. 19 (1990), 51-55.

[47] A. Tarski, Über einige fundamentale Begriffe der Metamathematik, C. R. Soc. Sci. Lettres Varsovie Cl. III 23 (1930), 22-29.

[48] A. Torrens, Model theory for sequential deductive systems, preprint, 1991.

[49] A. Torrens and V. Verdú, Abstract Eukasiewicz logics, preprint, 1990.

[50] V. Verdú, Contribution to the study of some classes of abstract logics, Ph.D. dissertation, Univ. of Barcelona, 1978 (in Catalan).

[51] —, Distributive and Boolean logics, Stochastica 3 (1979), 97-108 (in Catalan).

[52] - Some algebraic structures determined by closure operators, Z. Math. Logik Grundlag. Math. 31 (1985), 275-278.

[53] -, On some relations between closure operators and congruences, preprint, 1986. 
[54] V. Verdú, Logics projectively generated from $[M]=\left(F_{4},[\{1\}]\right)$ by a set of homomorphisms, Z. Math. Logik Grundlag. Math. 33 (1987), 235-241.

[55] R. Wójcicki, Lectures on Propositional Calculi, Ossolineum, Wrocław 1984.

[56] - , Theory of Logical Calculi. Basic Theory of Consequence Operations, Synthese Library 199, Reidel, Dordrecht 1988. 